\title{
Synthesis of Triptycenemonohydroquinonedibenzoquinone by
}

\section{Comproportionation}

Yuta Takemasa and Kyoko Nozaki*

Department of Chemistry and Biotechnology, Graduate School of Engineering, The University of Tokyo, 7-3-1 Hongo, Bunkyo-ku, Tokyo 113-8656, Japan.

Email: nozaki@chembio.t.u-tokyo.ac.jp

$\begin{array}{ll}\text { Content } & \text { S1 }\end{array}$

${ }^{1} \mathrm{H}$ NMR and ${ }^{13} \mathrm{C}$ NMR spectra for all compounds and reactions $\quad$ S2

UV-Vis spectra for all compounds and reactions $\quad$ S7

$\begin{array}{ll}\text { DFT calculation for }(\mathrm{HQ})_{1}(\mathrm{BQ})_{2} & \mathrm{~S} 8\end{array}$

Detail for the single crystal X-ray diffraction analysis for all compounds S9

References

$\mathrm{S} 13$ 
NMR Spectra for all compounds and reactions

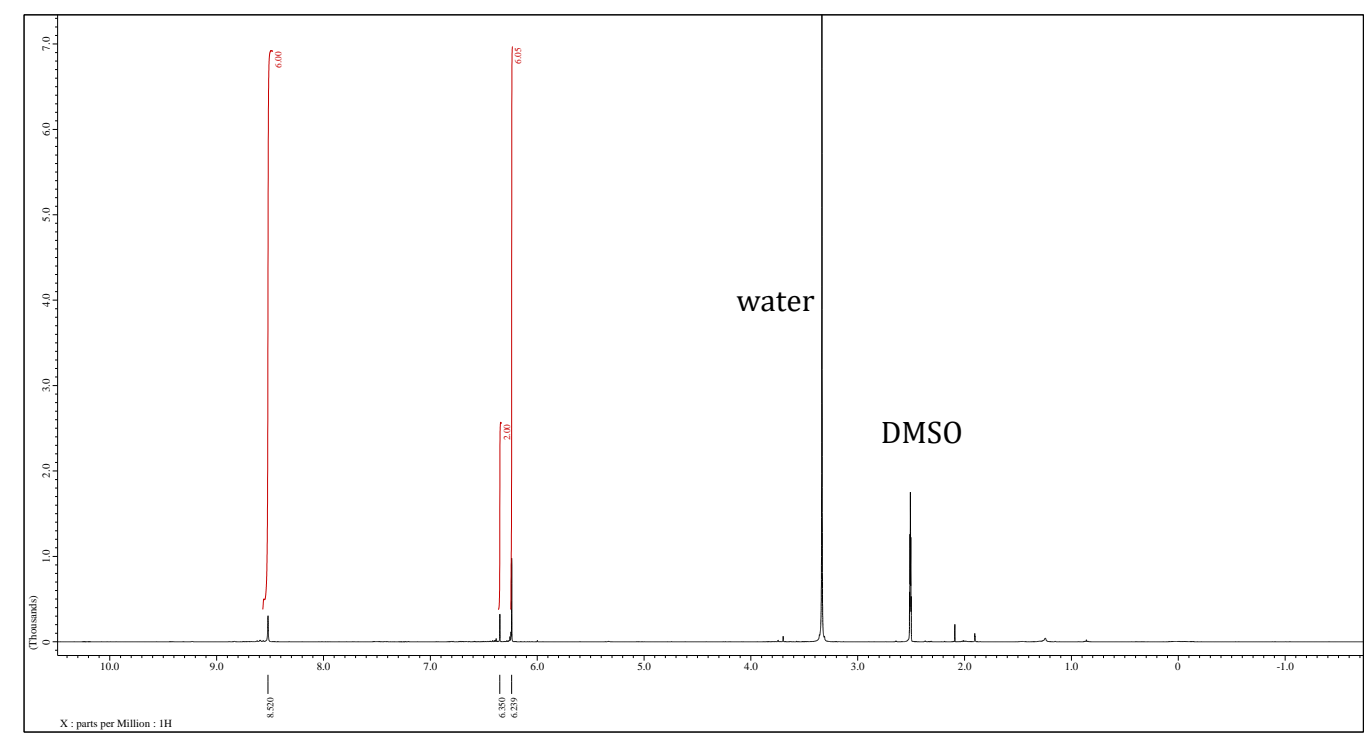

Figure S1. ${ }^{1} \mathrm{H}$ NMR spectrum $\left(500 \mathrm{MHz}\right.$, DMSO- $\left.d_{6}\right)$ of $(\mathrm{HQ})_{3}$

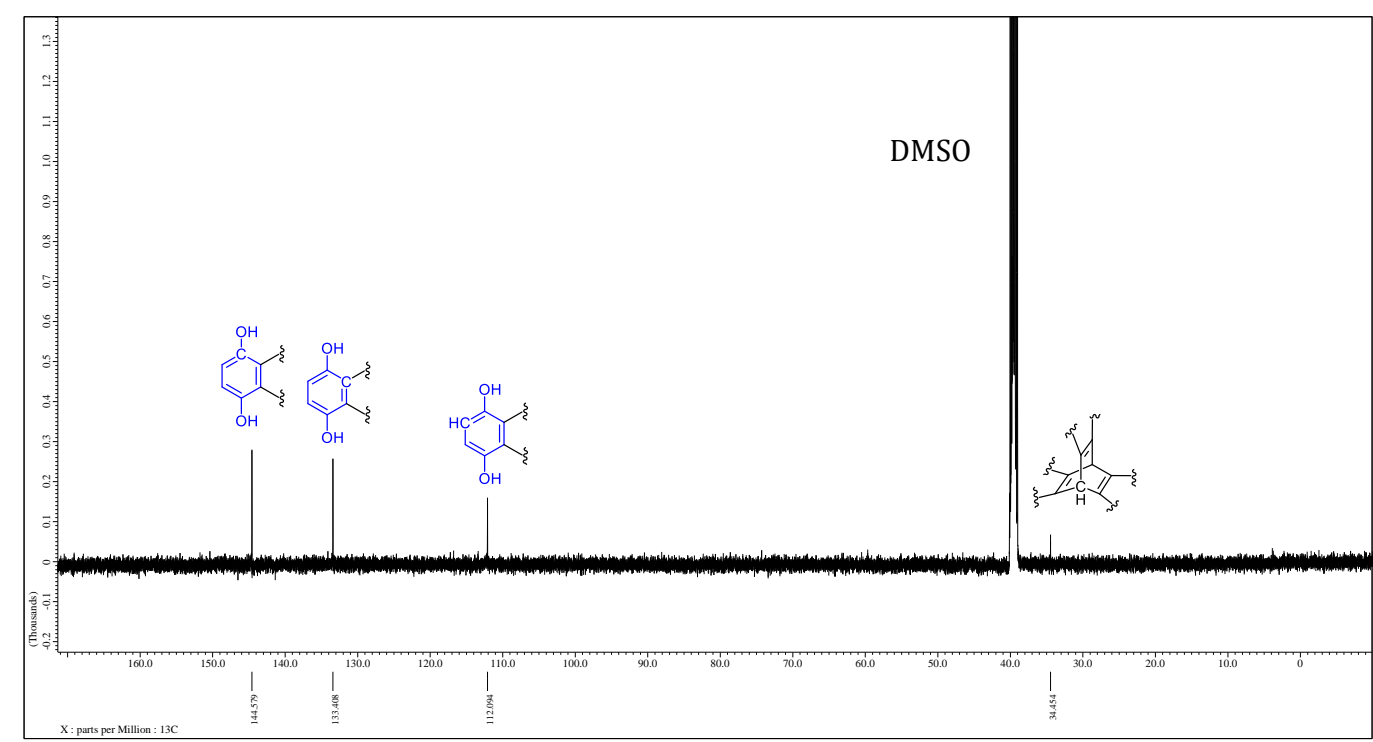

Figure $\mathrm{S} 2 .{ }^{13} \mathrm{C}\left\{{ }^{1} \mathrm{H}\right\}$ NMR spectrum $\left(126 \mathrm{MHz}\right.$, DMSO-d $d_{6}$ of $(\mathrm{HQ})_{3}$ 

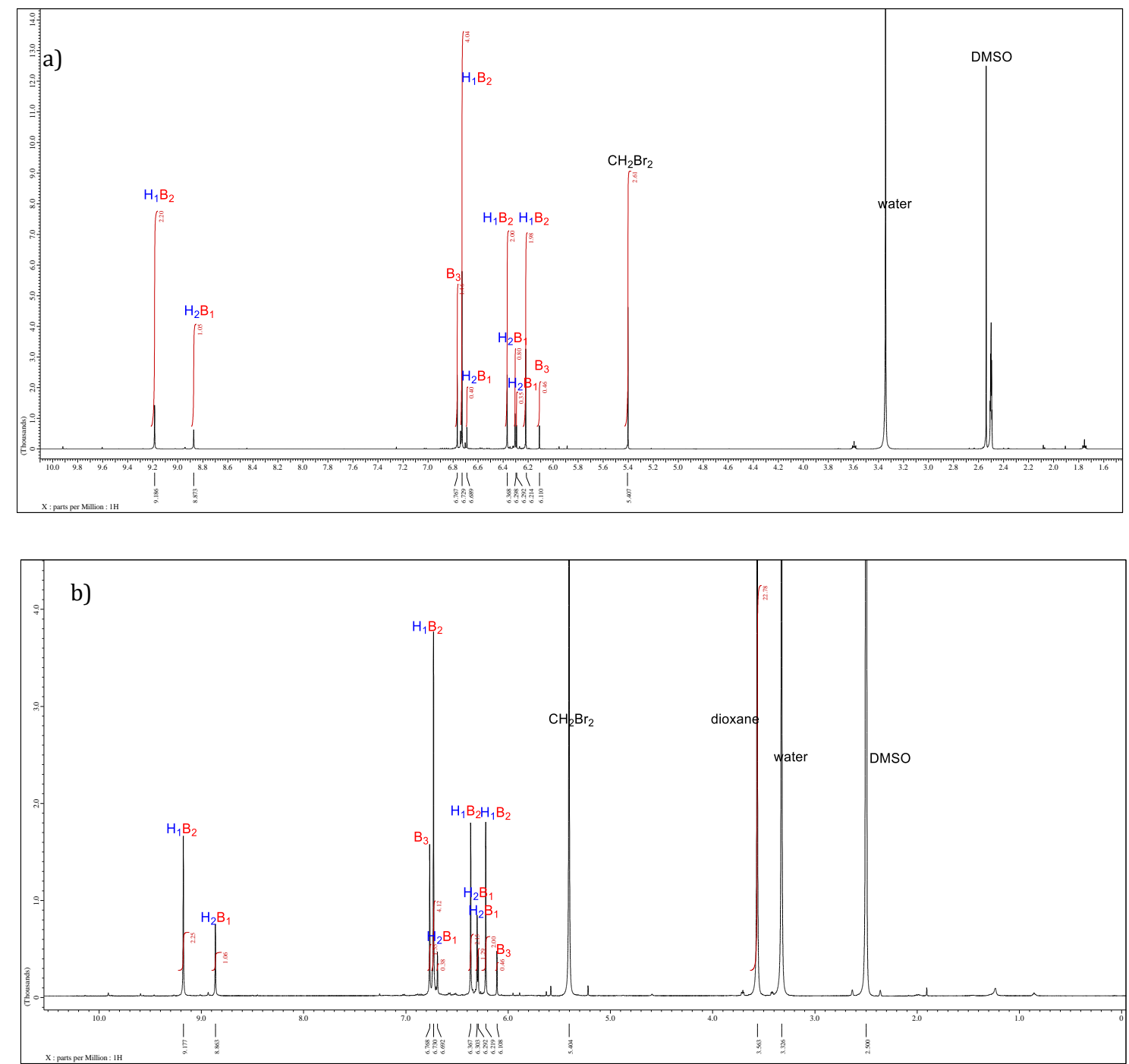

Figure S3. ${ }^{1} \mathrm{H}$ NMR spectra $\left(500 \mathrm{MHz}\right.$, DMSO- $d_{6}$ ) of (a) the reaction mixture in comproportionation of $(\mathrm{BQ})_{3}$ and $(\mathrm{HQ})_{3}(\mathrm{~b})$ disproportionation of $(\mathrm{HQ})_{1}(\mathrm{BQ})_{2} \cdot \mathrm{DMSO} \cdot 1.5$ dioxane (full charts of Figure 3 in the main text). $\left(\mathrm{B}_{3}=\right.$ $(B Q)_{3}, \mathrm{H}_{1} \mathrm{~B}_{2}=(\mathrm{HQ})_{1}(\mathrm{BQ})_{2}, \mathrm{H}_{2} \mathrm{~B}_{1}=(\mathrm{HQ})_{2}(\mathrm{BQ})_{1}$.) $\mathrm{CH}_{2} \mathrm{Br}_{2}$ : internal standard 

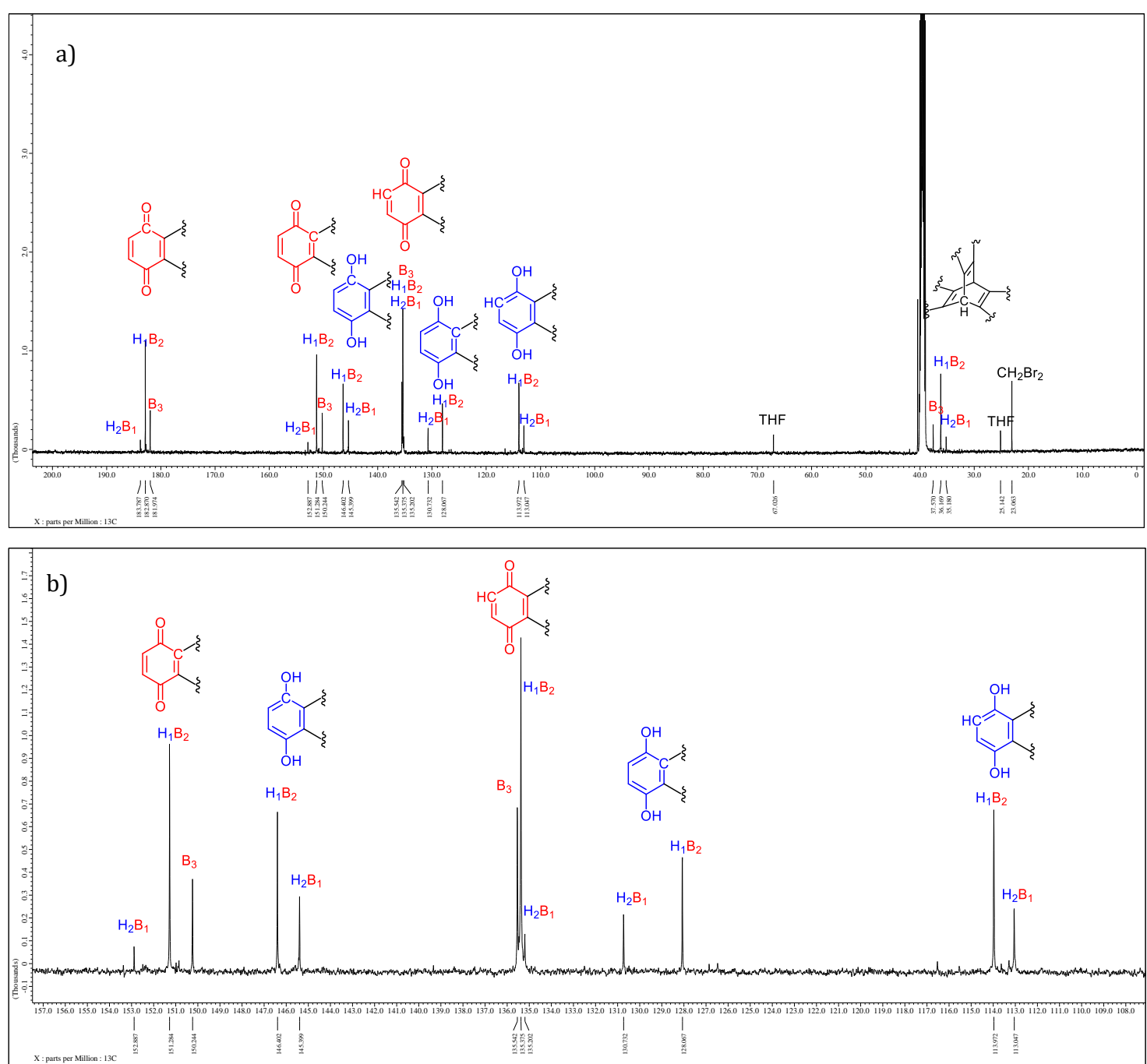

Figure S4 ${ }^{13} \mathrm{C}\left\{{ }^{1} \mathrm{H}\right\}$ NMR spectrum $\left(126 \mathrm{MHz}\right.$, DMSO- $\left.d_{6}\right)$ of (a) the reaction mixture in comproportionation of $(B Q)_{3}$ and $(H Q)_{3}$ (same sample as Figure 3) and (b) magnification $\left(\mathrm{H}_{1} \mathrm{~B}_{2}=(\mathrm{HQ})_{1}(\mathrm{BQ})_{2}, \mathrm{H}_{2} \mathrm{~B}_{1}=(\mathrm{HQ})_{2}(\mathrm{BQ})_{1}\right.$, $\mathrm{B}_{3}=(\mathrm{BQ})_{3}$.) $\mathrm{CH}_{2} \mathrm{Br}_{2}$ : internal standard 

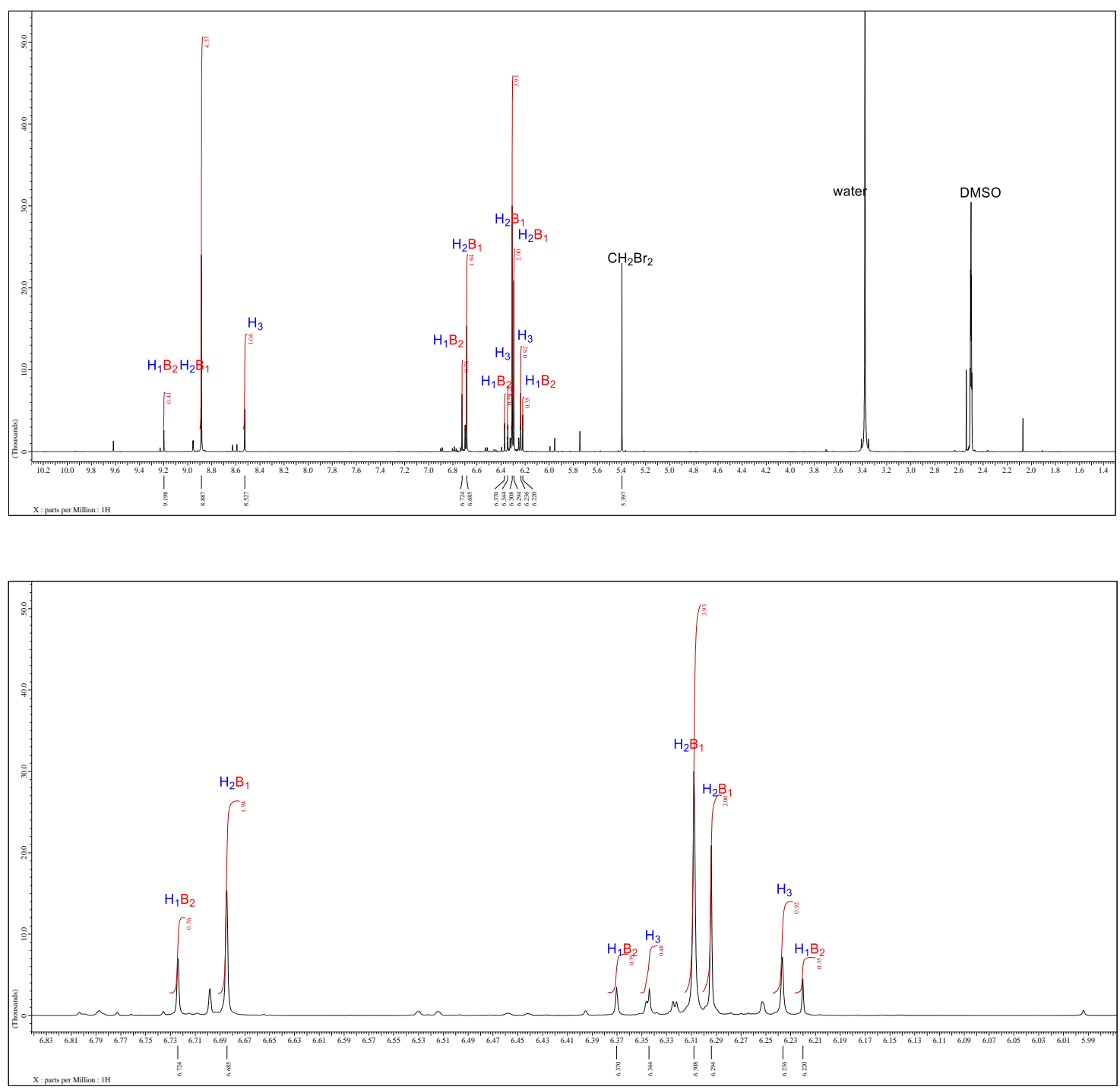

Figure S5. ${ }^{1} \mathrm{H}$ NMR spectrum $\left(500 \mathrm{MHz}\right.$, DMSO- $\left.d_{6}\right)$ of a) the reaction mixture in comproportionation of $(\mathrm{HQ})_{3}$ and $(B Q)_{3}$ in 2.0: 1.0 ratio and b) magnification. $\left(\mathrm{H}_{1} \mathrm{~B}_{2}=(\mathrm{HQ})_{1}(\mathrm{BQ})_{2}, \mathrm{H}_{2} \mathrm{~B}_{1}=(\mathrm{HQ})_{2}(\mathrm{BQ})_{1}, \mathrm{H}_{3}=(\mathrm{HQ})_{3}\right.$.) $\mathrm{CH}_{2} \mathrm{Br}_{2}$ : internal standard 

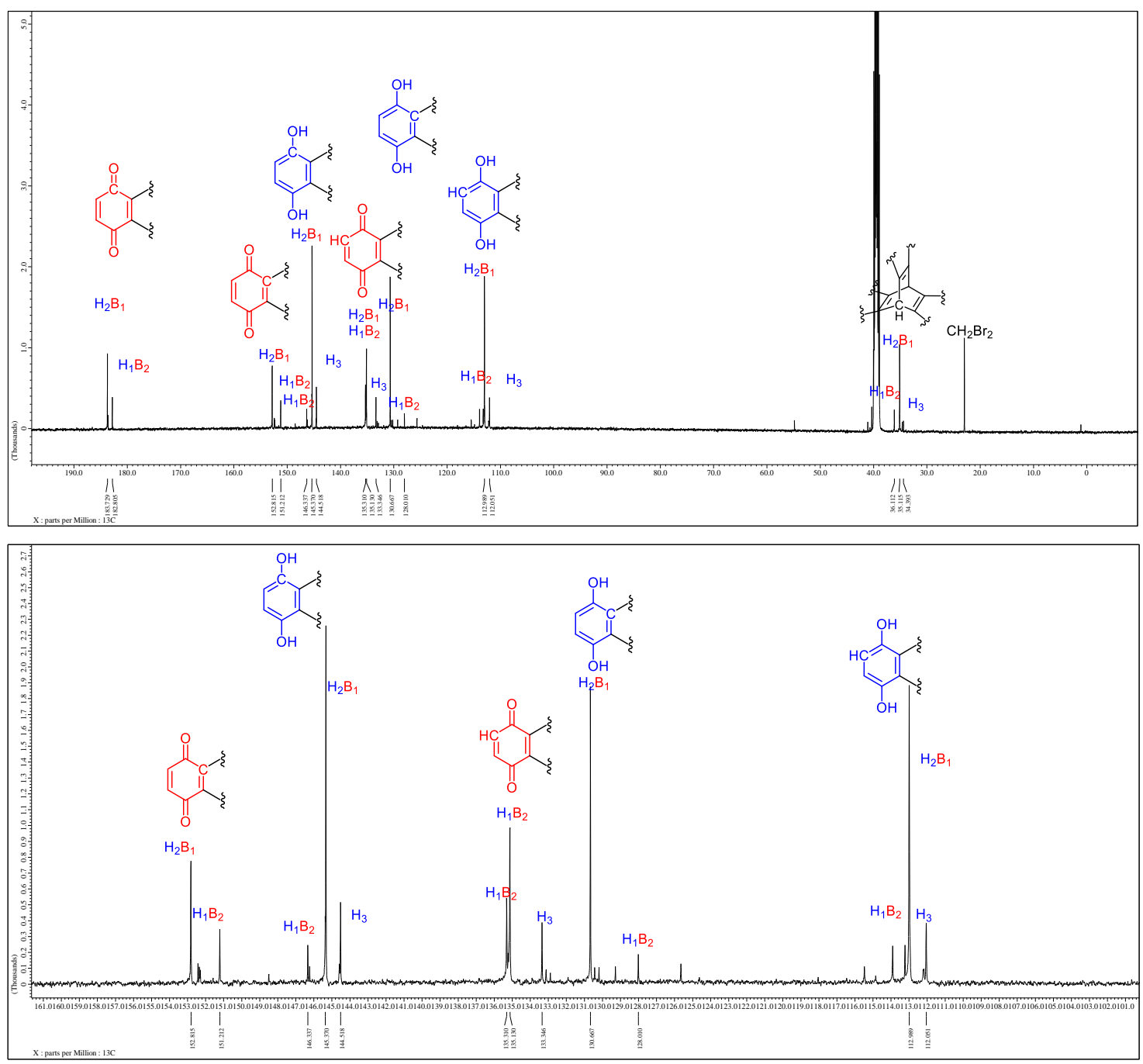

Figure S6. ${ }^{13} \mathrm{C}\left\{{ }^{1} \mathrm{H}\right\}$ NMR spectrum (126 MHz, DMSO- $d_{6}$ ) of a) the reaction mixture in comproportionation of $(\mathrm{HQ})_{3}$ and $(B Q)_{3}$ in 2.0: 1.0 ratio and b) magnification. $\left(\mathrm{H}_{1} \mathrm{~B}_{2}=(\mathrm{HQ})_{1}(\mathrm{BQ})_{2}, \mathrm{H}_{2} \mathrm{~B}_{1}=(\mathrm{HQ})_{2}(\mathrm{BQ})_{1}, \mathrm{H}_{3}=\left(\mathrm{HQ}_{3}\right.\right.$.) $\mathrm{CH}_{2} \mathrm{Br}_{2}$ : internal standard 


\section{UV-Vis Spectra for all compounds and reactions}

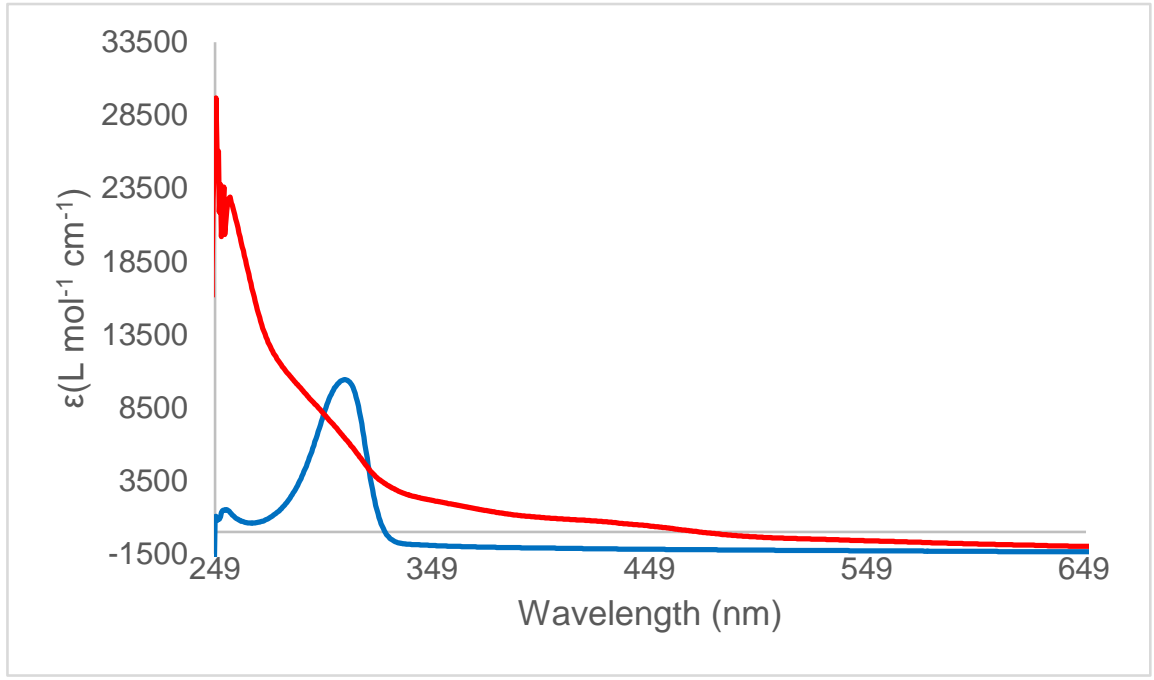

Figure S7. UV-Vis spectra of $(\mathrm{HQ})_{3}$ (blue) and $(\mathrm{BQ})_{3}(\mathrm{red})\left(\mathrm{DMSO}, 1.0 \times 10^{-4} \mathrm{M}\right)$

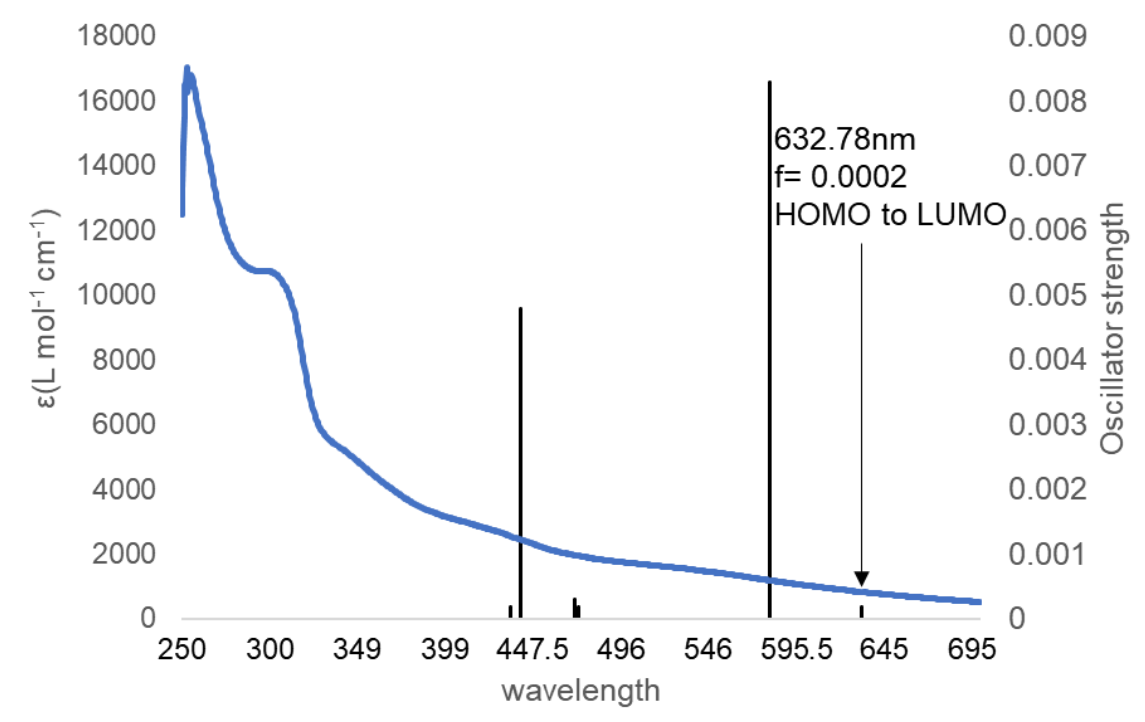

Figure S8. UV-Vis spectrum of reaction mixture of comproportionation 1week after admixture(blue) (DMSO, 1.0 $\times 10^{-4} \mathrm{M}$ ) and calculated spectrum of $(\mathrm{HQ})_{1}(\mathrm{BQ})_{2}$ by TD-DFT at the M06/6-31G(d) level (black). 


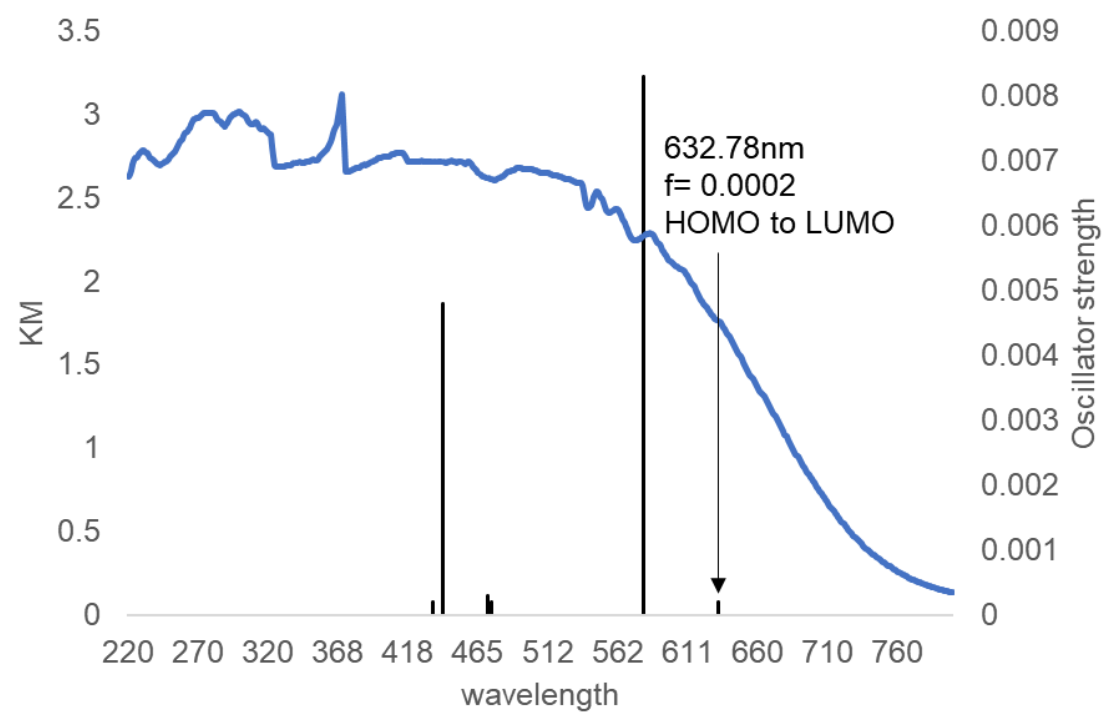

Figure S9. UV-Vis spectrum of crystal $(\mathrm{HQ})_{1}(\mathrm{BQ})_{2} / \mathrm{DMSO} /$ dioxane in solid state (blue) and calculated spectrum of $(\mathrm{HQ})_{1}(\mathrm{BQ})_{2}$ by TD-DFT at the M06/6-31G(d) level (black)

\section{DFT calculation for $(\mathrm{HQ})_{1}(\mathrm{BQ})_{2}$}

DFT calculations were performed using workstation at Research Center for Computational Science, National Institutes of Natural Sciences, Okazaki, Japan. All the calculations were performed by using Gaussian 09 Program, revision E.01.3.

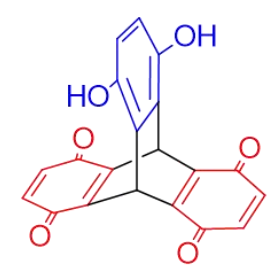

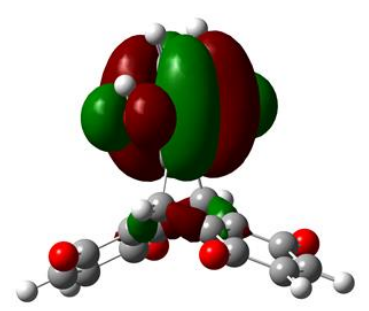

HOMO -6.10 eV

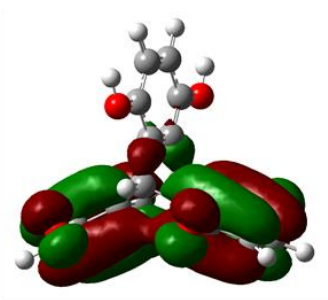

LUMO $-3.29 \mathrm{eV}$

Figure S10. Molecular orbitals (HQ) $1(\mathrm{BQ})_{2}$ at the M06/6-31G(d) level.

Based on the calculated molecular orbital structures, intramolecular charge transfer complex is less likely. On the other hand, in UV-Vis spectra, a broad signal at longer wave-length region than HOMO-LUMO gap was observed for the mixture of comproportionation (in solution) and crystals (in solid). From this observation, some sort of intermolecular interaction is implied. 
Detail for the single crystal $X$-ray diffraction analysis for all compounds

Table. S1 Crystallographic Data

\begin{tabular}{|c|c|c|c|c|}
\hline & $(\mathrm{HQ})_{3} / \mathrm{DMSO}$ & $\begin{array}{l}(\mathrm{HQ})_{1}(\mathrm{BQ})_{2} / \\
\text { DMSO/dioxane }\end{array}$ & $\begin{array}{l}(\mathrm{HQ})_{1}(\mathrm{BQ})_{2} / \\
\mathrm{H}_{2} \mathrm{O} / \text { dioxane }\end{array}$ & $(B Q)_{3}$ \\
\hline CCDC number & 2079417 & 2079383 & 2079384 & 1574417 \\
\hline Empirical formula & $\mathrm{C}_{22} \mathrm{H}_{10} \mathrm{O}_{7} \mathrm{~S}_{1}$ & $\mathrm{C}_{28} \mathrm{H}_{28} \mathrm{O}_{10} \mathrm{~S}_{1}$ & $\mathrm{C}_{28} \mathrm{H}_{28} \mathrm{O}_{11}$ & $\mathrm{C}_{20} \mathrm{H}_{8} \mathrm{O}_{6}$ \\
\hline Formula weight & 428.46 & 556.58 & 540.52 & 344.28 \\
\hline Temperature (K) & 93 & 93 & 93 & 93 \\
\hline Wavelength $(\AA ̊)$ & 0.71073 & 0.71073 & 0.71073 & 0.71073 \\
\hline Crystal system & monoclinic & triclinic & triclinic & tetragonal \\
\hline Space group & $C 2 / \mathrm{c}$ & $P-1$ & $\mathrm{P}-1$ & $I 4{ }_{1} \mathrm{md}$ \\
\hline \multicolumn{5}{|l|}{ Unit cell dimensions } \\
\hline a $(\AA ̊)$ & $22.2021(13)$ & $8.1826(5)$ & $9.2014(3)$ & $10.7619(3)$ \\
\hline $\mathrm{b}(\AA)$ & $7.8711(4)$ & $10.3792(6)$ & $9.8937(3)$ & $10.7619(3)$ \\
\hline c $(\AA)$ & $13.8109(9)$ & $16.0983(7)$ & $12.9678(3)$ & $12.6921(6)$ \\
\hline$\alpha\left(^{\circ}\right)$ & 90 & $94.678(4)$ & $79.274(2)$ & 90 \\
\hline$\beta\left({ }^{\circ}\right)$ & $93.763(5)$ & $97.331(4)$ & $78.371(2)$ & 90 \\
\hline$\gamma\left({ }^{\circ}\right)$ & 90 & $105.822(5)$ & $80.340(2)$ & 90 \\
\hline Volume $\left(\AA^{3}\right)$ & $2408.3(2)$ & $1294.92(13)$ & $1125.64(6)$ & $1469.98(11)$ \\
\hline $\mathrm{Z}$ & 8 & 1 & 2 & 16 \\
\hline Density (calculated) $\left(\mathrm{g} / \mathrm{m}^{3}\right)$ & 1.397 & 1.427 & 1.465 & 1.556 \\
\hline Absorption coefficient $\left(\mathrm{mm}^{-1}\right)$ & 0.268 & 0.185 & 0.114 & 0.987 \\
\hline$F(000)$ & 1064 & 584 & 520 & 704 \\
\hline Theta (max) & 29.213 & 27.877 & 28.875 & 71.874 \\
\hline \multirow[t]{3}{*}{ Index ranges } & $-28<\mathrm{h}<27$ & $-9<\mathrm{h}<9$ & $-12<\mathrm{h}<10$ & $-11<\mathrm{h}<6$ \\
\hline & $-10<\mathrm{k}<10$ & $-12<\mathrm{k}<12$ & $-13<\mathrm{k}<13$ & $-12<\mathrm{k}<13$ \\
\hline & $-18<\mathrm{l}<17$ & $-18<\mathrm{l}<17$ & $-17<\mathrm{l}<16$ & $-11<\mathrm{l}<15$ \\
\hline Reflections collected & 11865 & 12934 & 15779 & 1773 \\
\hline Independent reflections & 2854 & 4578 & 5222 & 599 \\
\hline$[\mathrm{R}(\mathrm{int})]$ & [0.0383] & [0.0815] & [0.0453] & [0.0573] \\
\hline
\end{tabular}




$\begin{array}{lllll}\text { Data Completeness (\%) } & 0.873 & 0.999 & 0.881 & 0.984 \\ \text { Absorption correction } & 2854 & 4578 & 5222 & 599 \\ \text { Refinement method } & \text { Multi-scan } & \text { Multi-scan } & \text { Multi-scan } & \text { Multi-scan } \\ \text { Data / restraints / parameters } & 2854 / 0 / 184 & 4578 / 0 / 356 & 5222 / 0 / 333 & 599 / 1 / 70 \\ \text { Goodness-of-fit on F } & 1.059 & 1.043 & 1.046 & 1.107 \\ & R_{1}=0.0755, & R_{1}=0.0978, & R_{1}=0.0496, & R_{1}=0.0651, \\ \text { Final R indices [I>2s(I)] } & w R_{2}=0.2071 & w R_{2}=0.1933 & w R_{2}=0.1154 & w R_{2}=0.1593 \\ & R 1=0.0710, & R_{1}=0.0725, & R_{1}=0.0424, & R_{1}=0.0640, \\ \text { R indices (all data) } & w R_{2}=0.2028 & w R_{2}=0.2092 & w R_{2}=0.1112 & w R_{2}=0.1581 \\ & 2.036 /-1.128 & 0.825 /-0.521 & 0.368 /-0.313 & 0.341 /-0.536\end{array}$

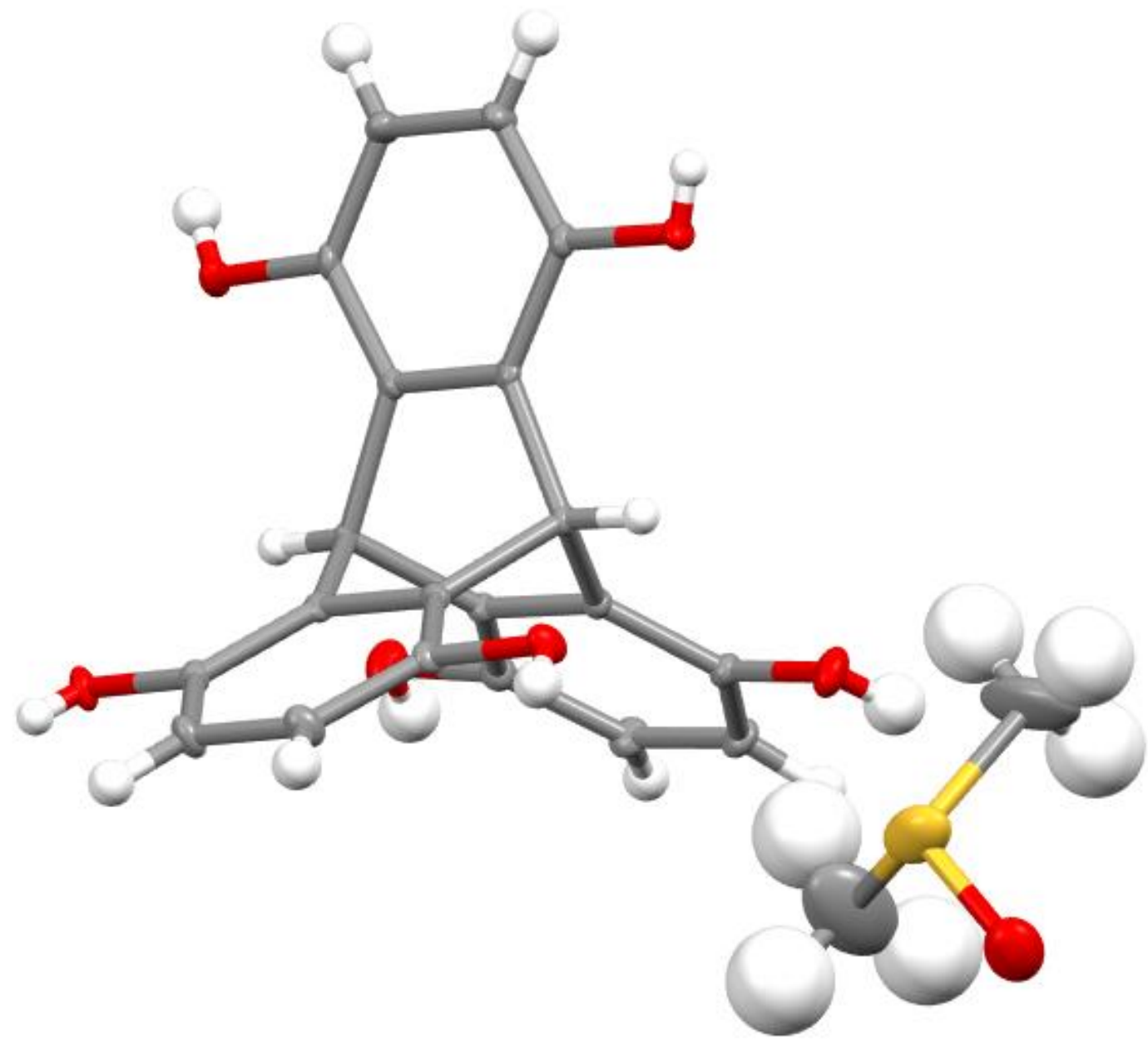

Figure S11. X-ray structure of (HQ) 3 . Thermal ellipsoids are drawn with 50\% probability. 


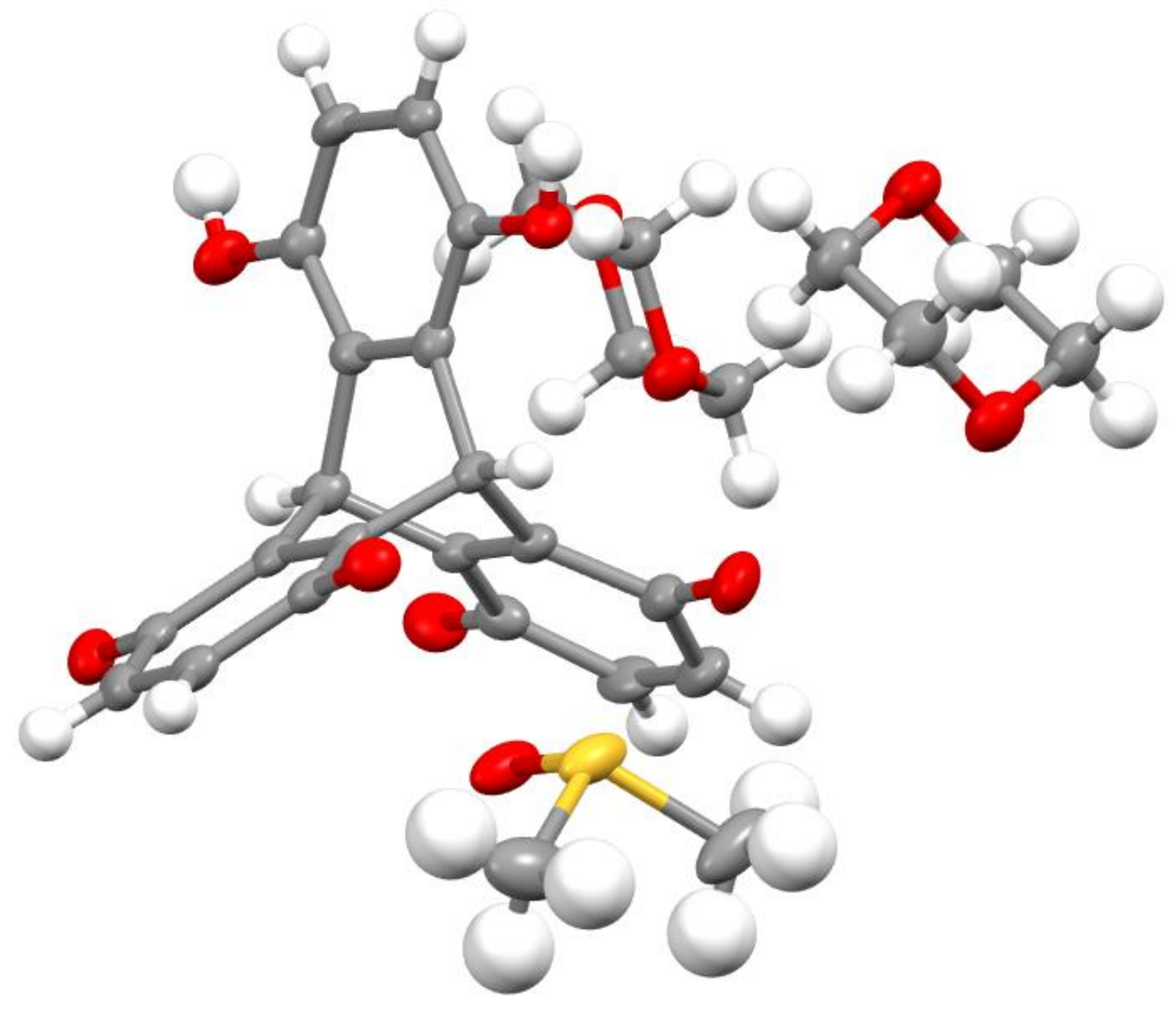

Figure S12. X-ray structure of $(\mathrm{HQ})_{1}(\mathrm{BQ})_{2} / \mathrm{DMSO} /$ dioxane. Thermal ellipsoids are drawn with 50\% probability. 


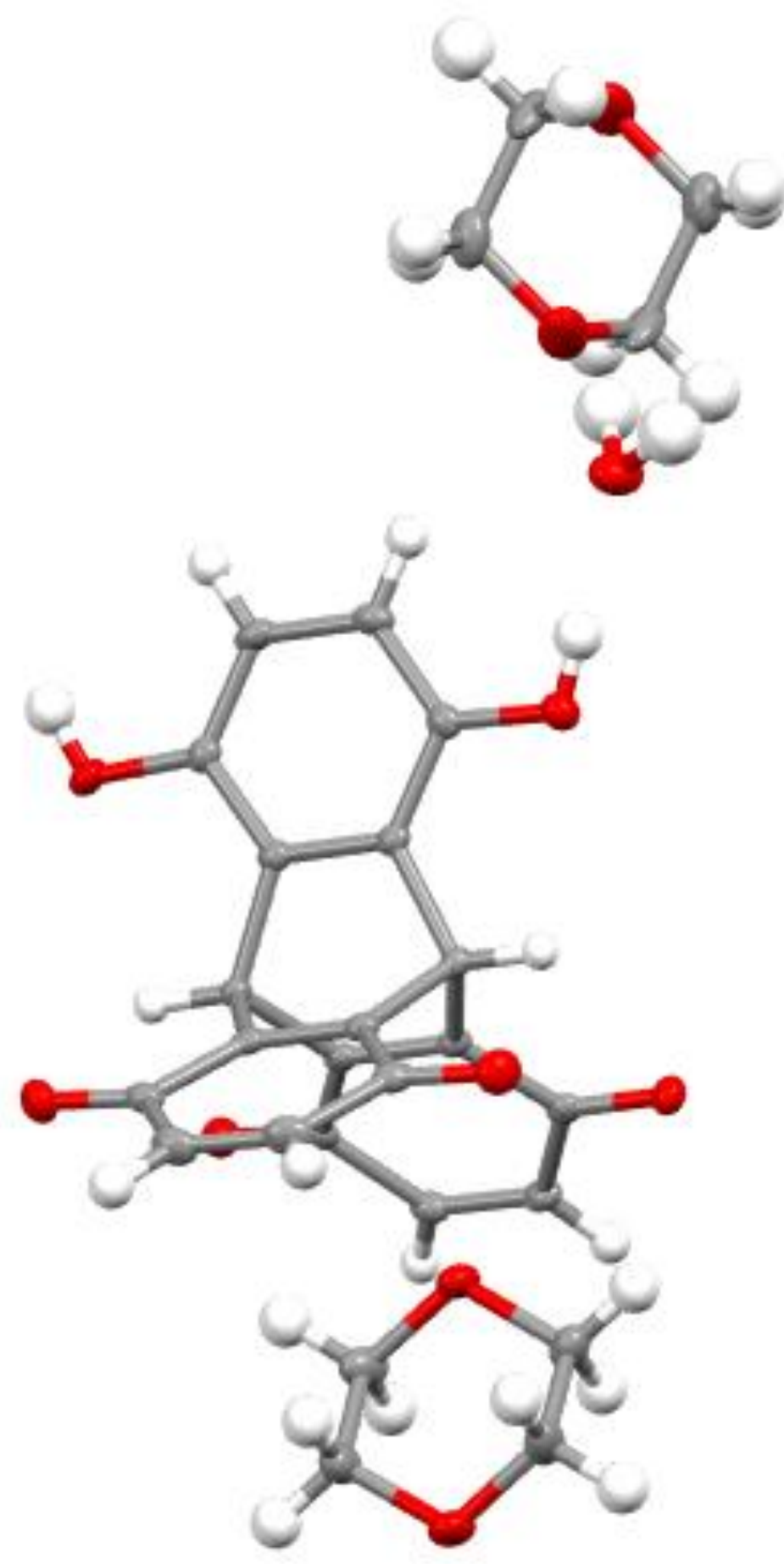

Figure S13. X-ray structure of $(\mathrm{HQ})_{1}(\mathrm{BQ})_{2} / \mathrm{H}_{2} \mathrm{O} /$ dioxane. Thermal ellipsoids are drawn with $50 \%$ probability. 


\section{References}

[1] Gaussian 09, Revision E.01, M. J. Frisch, G. W. Trucks, H. B. Schlegel, G. E. Scuseria, M. A. Robb, J. R.

Cheeseman, G. Scalmani, V. Barone, B. Mennucci, G. A. Petersson, H. Nakatsuji, M. Caricato, X. Li, H. P. Hratchian, A. F. Izmaylov, J. Bloino, G. Zheng, J. L. Sonnenberg, M. Hada, M. Ehara, K. Toyota, R. Fukuda, J. Hasegawa, M. Ishida, T. Nakajima, Y. Honda, O. Kitao, H. Nakai, T. Vreven, J. A. Montgomery, Jr., J. E. Peralta, F. Ogliaro, M. Bearpark, J. J. Heyd, E. Brothers, K. N. Kudin, V. N. Staroverov, T. Keith, R. Kobayashi, J. Normand, K. Raghavachari, A. Rendell, J. C. Burant, S. S. Iyengar, J. Tomasi, M. Cossi, N. Rega, J. M. Millam, M. Klene, J. E. Knox, J. B. Cross, V. Bakken, C. Adamo, J. Jaramillo, R. Gomperts, R. E. Stratmann, O. Yazyev, A. J. Austin, R. Cammi, C. Pomelli, J. W. Ochterski, R. L. Martin, K. Morokuma, V. G. Zakrzewski, G. A. Voth, P. Salvador, J. J. Dannenberg, S. Dapprich, A. D. Daniels, O. Farkas, J. B. Foresman, J. V. Ortiz, J. Cioslowski, D. J. Fox, Gaussian 09, Revision E.01, Gaussian, Inc., Wallingford CT, 2013. 